\title{
Optimal acupoint and session of acupuncture for patients with chronic prostatitis/chronic pelvic pain syndrome: a meta-analysis
}

\author{
Wei Zhang ${ }^{1 \#}$, Yu Fang ${ }^{1 \#}$, Minfeng Shi ${ }^{2}$, Mingzhen Zhang $^{3,4}$, Yuangui Chen ${ }^{5}$, Tie Zhou ${ }^{1}$ \\ ${ }^{1}$ Department of Urology, Changhai Hospital, Naval Medical University, Shanghai, China; ${ }^{2}$ Reproductive Center, Changhai Hospital, Naval Medical \\ University, Shanghai, China; ${ }^{3}$ Second Affiliated Hospital and Yuying Children's Hospital, Wenzhou Medical University, Wenzhou, China; ${ }^{4}$ WMU- \\ Monash University BDI Alliance in Clinical \& Experimental Biomedicine, Wenzhou Medical University, Wenzhou, China; ${ }^{5}$ Hongkou Branch, \\ Changhai Hospital, Naval Medical University, Shanghai, China \\ Contributions: (I) Conception and design: T Zhou, Y Chen, W Zhang; (II) Administrative support: T Zhou, Y Chen; (III) Provision of study materials \\ or patients: W Zhang, Y Fang, M Shi; (IV) Collection and assembly of data: W Zhang, Y Fang; (V) Data analysis and interpretation: W Zhang, Y \\ Fang, M Shi, M Zhang; (VI) Manuscript writing: All authors; (VII) Final approval of manuscript: All authors. \\ \#These authors contributed equally to this work. \\ Correspondence to: Tie Zhou, MD, PhD. Department of Urology, Changhai Hospital, Naval Medical University, 168 Changhai Rd, Shanghai 200433 , \\ China. Email: wenzhoutie@163.com; Yuangui Chen, MD. Hongkou Branch, Changhai Hospital, Naval Medical University, 15 East Jiangwan Rd, \\ Shanghai 200081, China. Email: orthodocchen@163.com.
}

Background: The study aims to perform a meta-analysis of published trials and evaluate the efficacy of acupuncture on chronic prostatitis/chronic pelvic pain syndrome (CP/CPPS) by symptom score reduction, optimal acupuncture session, and most frequently used acupoints.

Methods: A literature search was performed for randomized controlled trials (RCTs) comparing efficacy of acupuncture with sham acupuncture or standard medication on CP/CPPS. The primary outcome was the reduction of National Institute of Health-Chronic Prostatitis Index (NIH-CPSI) total score and its subscales. The optimal acupuncture session to reach its clinical efficacy and most common compatibility rule of acupoints were also evaluated.

Results: Ten trials involving 770 participants were included. Meta-analysis showed compared with sham acupuncture, acupuncture yielded significant reduction in NIH-CPSI total score [weighted mean difference (WMD): 7.28, 95\% confidence interval (95\% CI): 5.69-8.86), and provided better pain relief (WMD: 3.57, 95\% CI: 2.07-5.08), urinary symptoms improvement (WMD: 1.68, 95\% CI: 1.13-2.22), and quality of life (QOL) (WMD: 2.38, 95\% CI: 1.41-3.36). Compared with standard medication, acupuncture were more efficacious in reducing NIH-CPSI total score (WMD: 3.36, 95\% CI: 1.27-5.45), also showed significant greater pain relief (WMD: 2.36, 95\% CI: 1.67-3.06), marginal advantage in improving QOL (WMD: 0.98, 95\% CI: $0.12-1.83$ ) but no difference in reducing urinary symptom (WMD: $-0.03,95 \%$ CI: -1.30 to 1.24 ). Four acupuncture sessions were the minimum "dose" to reach clinical efficacy, and prolonged acupuncture sessions continuously improved urinary symptoms and QOL. The majority of acupoint selection strategies were based on the combination of any three acupoints from CV3, CV4, BL32, SP6, and SP9.

Conclusions: Acupuncture has promising efficacy for patients with CP/CPPS, especially category IIIB, in aspects of relieving pain and urinary symptoms and improving the QOL. Acupuncture may serve as a standard treatment option when available, and a tailored comprehensive treatment strategy for CP/CPPS is the future trend.

Keywords: Acupuncture; chronic prostatitis/chronic pelvic pain syndrome (CP/CPPS); National Institute of Health-Chronic Prostatitis Index (NIH-CPSI); acupuncture session; acupoint selection

Submitted May 06, 2020. Accepted for publication Oct 19, 2020.

doi: $10.21037 /$ tau-20-913

View this article at: http://dx.doi.org/10.21037/tau-20-913 


\section{Introduction}

Chronic prostatitis/chronic pelvic pain syndrome (CP/ CPPS) is defined as chronic pelvic pain lasting for at least 3 months, often associated with lower urinary tract symptoms and/or sexual dysfunction (1). Based on the classification by National Institutes of Health Prostatitis Collaborative Network, CP/CPPS is referred to category III prostatitis, and further subdivided into category IIIA and IIIB, with or without leukocytes in expressed prostatic secretions (2). CP/CPPS affects $2 \%$ to $15 \%$ of adult men, and it is associated with negative psychological effects and substantial health care costs $(3,4)$. Since the etiology of CP/ CPPS is multifactorial, various treatments including alphablockers, antibiotics, and nonsteroidal anti-inflammatory drugs are optional, whereas none of which has proven to be effective for any patient (5). Several alternative therapies such as diet and lifestyle modifications, phytotherapy, and myofascial physical therapy have controversial benefits (6).

As an important component of traditional Chinese medicine, acupuncture is one of the most rigorously examined alternative medication in many countries (7). It was reported that the efficacy of acupuncture on CP/ CPPS included anti-inflammatory, neural and immune modulation (8). With the gaining acceptance in application of acupuncture in urology, more and more high quality randomized controlled trials (RCTs) were available in the last 3 years (9-11). We aim to perform a meta-analysis to evaluate the efficacy of acupuncture on CP/CPPS by National Institutes of Health Chronic Prostatitis Symptom Index (NIH-CPSI) score reduction, and a meta-regression analysis of dose-response association between acupuncture sessions and treatment effect. We present the following article in accordance with the Preferred Reporting Items for Systematic Reviews and Meta-Analyses statement (PRISMA) reporting checklist (available at http://dx.doi. org/10.21037/tau-20-913).

\section{Methods}

\section{Search strategy}

A literature search was performed on February14, 2020, using PubMed, Embase, Web of Science, Wanfang Data, and China National Knowledge Infrastructure (CNKI) databases to identify relevant studies. The following keywords were used as search terms: (acupuncture OR electroacupuncture) AND (chronic prostatitis OR nonbacterial prostatitis OR chronic pelvic pain). Searches were limited to RCTs published in English and Chinese. Reference lists of the published systematic reviews were scanned as well. The retrieved articles were viewed by $W$ Zhang and Y Fang independently, and all disagreements were resolved by their consensus.

\section{Eligibility criteria}

RCTs that met following criteria were included: (I) participants whose age $\leq 50$ years were diagnosed with CP/CPPS (category IIIA or IIIB). (II) Trials reporting one of the following interventions: acupuncture $v s$. sham acupuncture, or acupuncture vs. standard medication, or acupuncture plus standard medication vs. standard medication, or acupuncture plus standard medication $v$ s. acupuncture alone. (III) The primary outcome utilizing the change of NIH-CPSI total score, and secondary outcomes including changes of NIH-CPSI subscales. The controlled clinical trials, cohort studies, and case reports were excluded.

\section{Quality and outcome assessment}

The methodological quality of eligible studies was evaluated according to the Jadad scale. The study with Jadad score $\geq 4$ means a high-quality trial, and with score $<4$ indicates a low-quality trial. The assessments were processed independently by two reviewers (W Zhang, Y Fang), and the final decision was determined by their discussion. The mean score changes with corresponding standard deviations (SEs) from baseline to endpoint of follow-up were needed to pool data. For those studies only reporting scores at the baseline and follow-up endpoint, the reduction changes of scores were estimated according to the previously published methodology (12). To perform a meta-analysis of continuous variables, the weighted mean differences (WMDs) with corresponding 95\% confidence intervals (CIs) were calculated. Furthermore, we illustrated the weighted mean reductions of NIH-CPSI score as effect estimators and the treatment sessions as "dose" in metaregression analysis of dose-response association between acupuncture sessions and acupuncture effects on CP/CPPS. Last, Apriori association analysis of acupoint selection was conducted to discover the most common compatibility rule of acupoints in included trials.

\section{Statistical analysis}


The Q statistic was used to test the heterogeneity among trials: homogeneity was rejected when the $\mathrm{Q}$ statistic $\mathrm{P}$ value was $<0.10$. Depending on whether homogeneity was accepted or rejected, we applied the fixed effect model or the random effect model to calculate the WMDs and 95\% CIs. Meta-analysis was conducted using Stata v.12.0 (StataCorp., College Station, TX, USA). Apriori association analysis was conducted using IBM SPSS Modeler v.14.1 (IBM, Armonk, NY, USA)

\section{Results}

\section{Study characteristics}

Ten manuscripts on RCTs for CP/CPPS were ultimately utilized for comparisons between acupuncture and sham acupuncture/standard medication as treatments for CP/ CPPS (Figure S1). The methodological quality of the included RCTs was median or high for all the analyzed trials (Jadad scale: 4-5 of 5 points). Four trials compared (electro-) acupuncture with sham (electro-)acupuncture (11,13-15), three trials compared (electro-)acupuncture with standard medication (16-18), one trial compared acupuncture with sham acupuncture/standard medication (19), and two trials compared acupuncture plus standard medication with acupuncture/standard medication alone $(20,21)$. Overall, 770 patients were included in meta-analysis. The characteristics and selected acupoints of included studies are respectively displayed in Tables S1,S2. The treatment time and sessions, selected acupoints, and follow-up periods varied among trials. All studies reported NIH-CPSI total scores and subdomain scores as their outcomes.

\section{Acupuncture vs. sham acupuncture}

\section{NIH-CPSI total score}

Among five trials involving 329 participants compared acupuncture to sham acupuncture, an average total NIHCPSI score reduction of 11.5 was observed in acupuncture group while 4.5 in sham acupuncture group (Figure 1). Meta-analysis showed that acupuncture yielded a significant reduction in NIH-CPSI total score compared with sham acupuncture (WMD: 7.28, 95\% CI: 5.69-8.86).

\section{NIH-CPSI subscales}

Meta-analysis showed that acupuncture was more efficacious than sham acupuncture in pain, urinary and quality of life (QOL) subscales. Acupuncture provided better pain relief (WMD: 3.57, 95\% CI: 2.07-5.08), urinary symptoms improvement (WMD: 1.68, 95\% CI: 1.13-2.22), and life quality (WMD: 2.38, 95\% CI: 1.41-3.36) than sham acupuncture.

\section{Acupuncture vs. standard medication}

\section{NIH-CPSI total score}

Five trials involving 346 participants compared acupuncture to standard medication, and found an average NIHCPSI total score reduction of 11.5 and 7.8 respectively in acupuncture and standard medication groups (Figure 2). Meta-analysis showed a more favorable effect of acupuncture on reducing NIH-CPSI total score compared with standard medication (WMD: 3.36, 95\% CI: 1.27-5.45).

\section{NIH-CPSI subscales}

Among the above five studies, four reported all the three subscales of NIH-CPSI score, whereas one only reported the pain subscale. Meta-analysis demonstrated that acupuncture significantly decreased pain subscale compared with standard medication (WMD: 2.36, 95\% CI: 1.67-3.06). However, only marginal difference in improving QOL subscale (WMD: 0.98, 95\% CI: 0.12-1.83) and no difference in reducing urinary subscale (WMD: $-0.03,95 \%$ CI: -1.30 to 1.24$)$ were observed between acupuncture and standard medication.

\section{Acupuncture plus standard medication vs. standard medication}

\section{NIH-CPSI total score}

Two trials involving 131 participants compared acupuncture plus standard medication to standard medication alone, reporting an average NIH-CPSI total score reduction of 11.8 in acupuncture plus standard medication group while 8.6 in standard medication group (Figure S2). Metaanalysis suggested that compared with standard medication alone, acupuncture plus standard medication resulted in significantly larger changes in NIH-CPSI total score (WMD: 3.20, 95\% CI: 1.97-4.43).

\section{NIH-CPSI subscales}

Among the above two studies, one reported all the three subscales of NIH-CPSI score, and another only reported the pain subscale. The pooled results of NIH-CPSI subscale reductions revealed that acupuncture plus standard medication provided better pain relief (WMD: 1.43, 95\% 


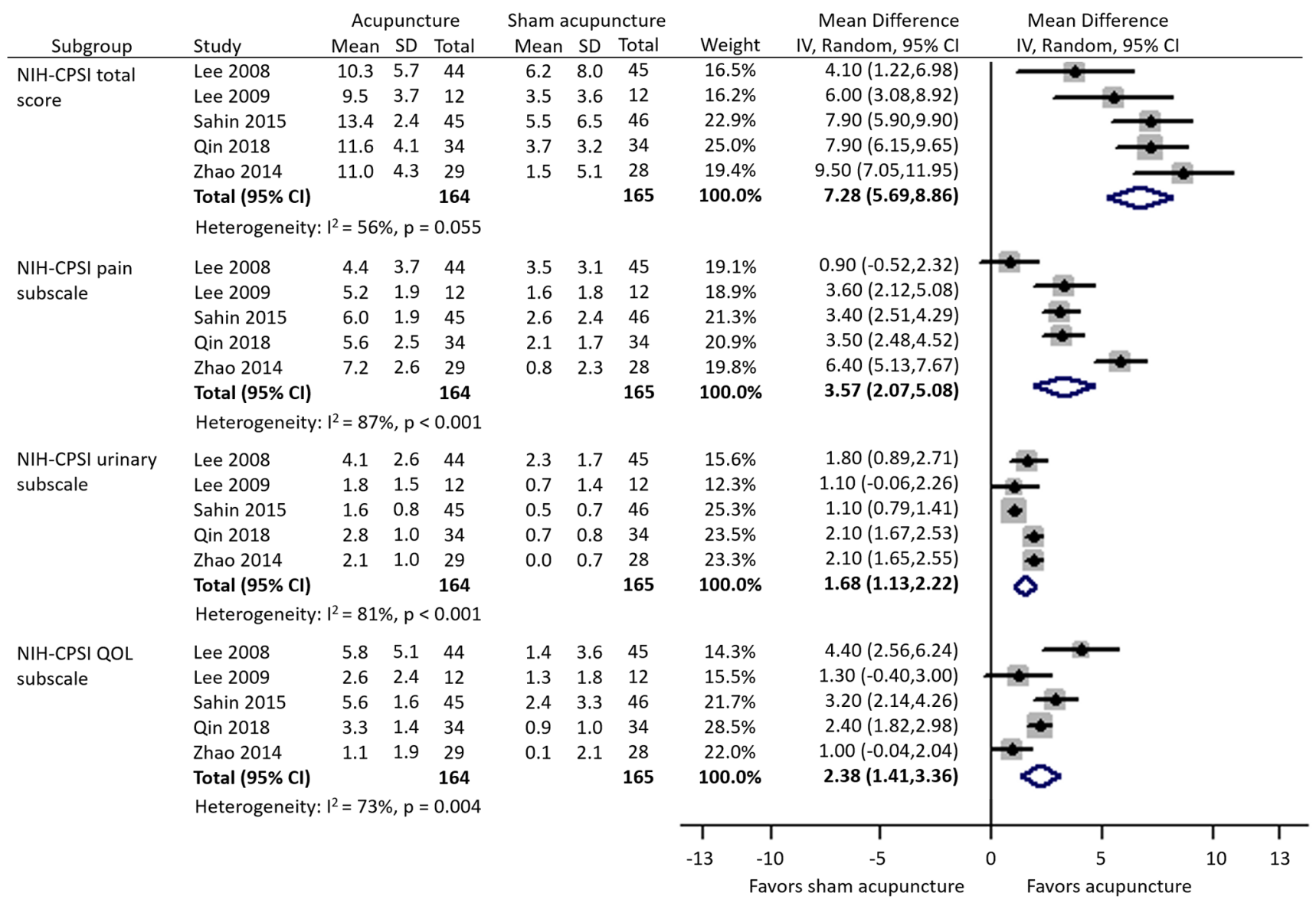

Figure 1 Forest plot of comparisons of NIH-CPSI score reduction between acupuncture and sham acupuncture groups. NIH-CPSI, National Institute of Health-Chronic Prostatitis Index.

CI: 0.80-2.07), but not urinary symptom (WMD: -0.10 , 95\% CI: -0.36 to 0.16 ) and life quality (WMD: $0.40,95 \%$ CI: -0.09 to 0.89$)$ improvements than standard medication alone.

\section{Acupuncture plus standard medication vs. acupuncture}

Only one trial involving 59 participants compared acupuncture plus standard medication to acupuncture alone (Figure S3). According to the reported NIH-CPSI data, acupuncture plus standard medication was preferred to acupuncture alone to reduce the NIH-CPSI total score (WMD: 2.60, 95\% CI: 1.07-4.13). However, the NIHCPSI pain subscale reduction did not show significant difference between these two groups (WMD: 0.70, 95\% CI: -1.01 to 2.41$)$.

\section{Acupuncture in category IILA + IIIB vs. IIIB}

Subgroup analyses were carried out according to the category of CP/CPPS (IIIA + IIIB vs. IIIB). For patients with category IIIB, there were two trials compared acupuncture to sham acupuncture, and three trials compared acupuncture to standard medication. Subgroup metaanalysis suggested that compared with sham acupuncture, acupuncture resulted in significantly larger changes in NIH-CPSI total score for patients with both category IIIB and IIIA + IIIB (WMD: $8.54 v s .6 .23)$. The efficacy advantages of relieving pain (WMD: 4.86 vs. 2.70) achieved by acupuncture for category IIIB were more significant than that for category IIIA + IIIB (Figure $3 A$ ). In addition, subgroup meta-analysis also showed that compared with medication, acupuncture significantly decreased NIH-CPSI 


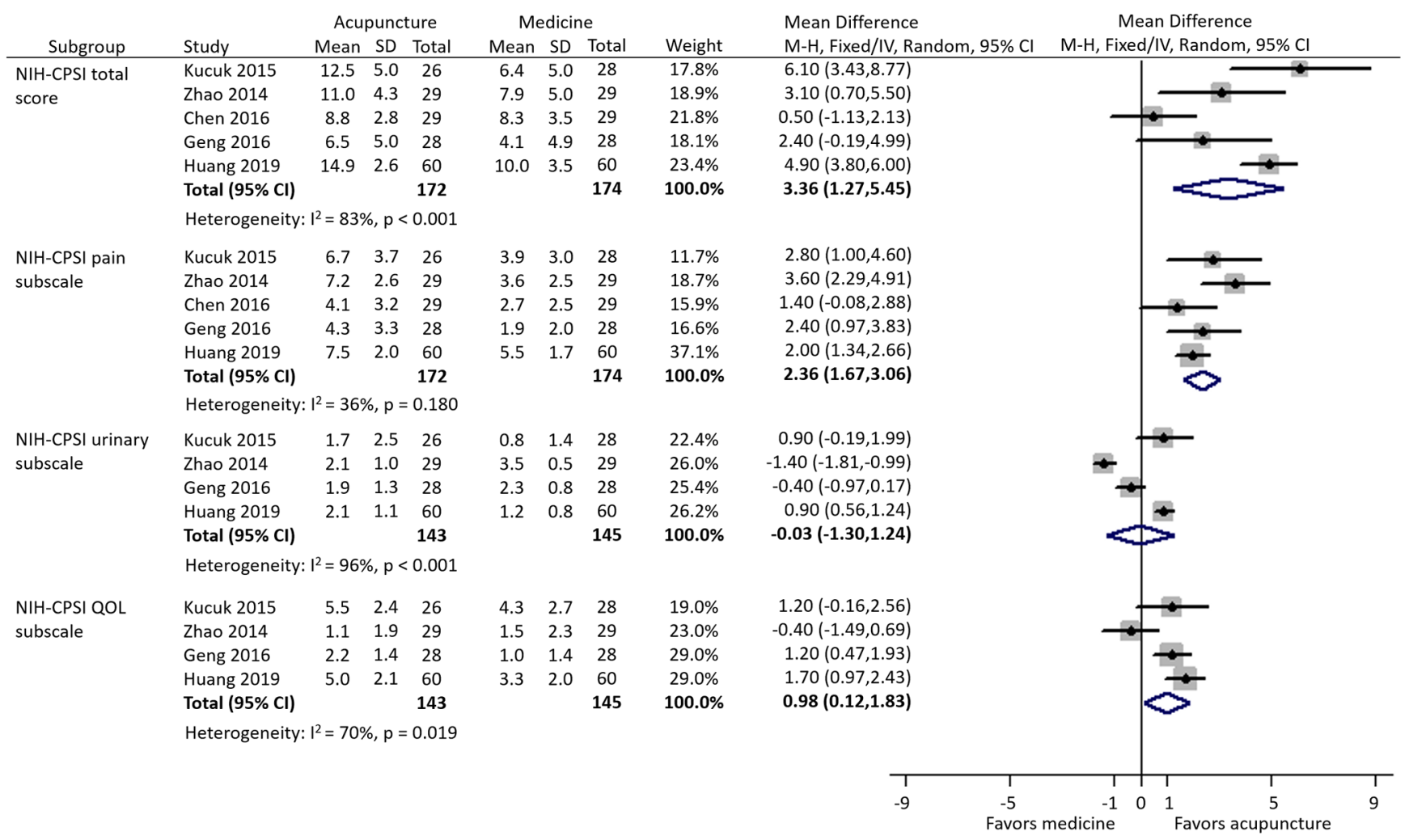

Figure 2 Forest plot of comparisons of NIH-CPSI score reduction between acupuncture and medication groups. NIH-CPSI, National Institute of Health-Chronic Prostatitis Index.

total score for patients with category IIIB (WMD: 3.83, 95\% CI: 1.67-5.99), but not for patients with category IIIA + IIIB (WMD: 2.74, 95\% CI: -1.57 to 7.05 ). The efficacy advantages of relieving pain (WMD: $2.99 v s .1 .90$ ) achieved by acupuncture for category IIIB were more significant than that for category IIIA + IIIB (Figure 3B).

\section{Association analysis of acupoint selection}

The average number of selected acupoints was 7 (range, 3-13) (Table S2). The five most frequently chosen acupoints were SP6 (Sanyinjiao), CV4 (Guanyuan), CV3 (Zhongji), BL32 (Ciliao), SP9 (Yinlingquan). The Apriori association analysis of acupoint selection demonstrated that the most common compatibility rule of two acupoints was the combination of SP6 and CV4, while the most frequently used compatibility rule of three acupoints was the combination of SP9, SP6 and CV4 (Figure 4A). In addition, the acupoint selection strategy of most trials was several other acupoints plus the combination of any three acupoints from SP6, CV4, CV3, BL32, and SP9 (Figure 4B).

\section{Acupuncture sessions and the NIH-CPSI score}

All of the ten trials were included in the meta-regression model between NIH-CPSI score reduction and acupuncture sessions. The result indicated an overall trend that more acupuncture sessions were associated with greater symptom relief. In detail, a J-shaped association between acupuncture sessions and NIH-CPSI total score was presented as follows (Figure 5A). First, after 4 acupuncture sessions, a NIH-CPSI score decline of 6 being regarded as the optimal threshold for minimal clinically important difference was achieved (22). Then, the NIH-CPSI score reached its maximum reduction of 12 after 16 acupuncture sessions. Finally, the reduction of NIH-CPSI score was fluctuating between 10 and 12 with continuing acupuncture sessions. The similar J-shaped regression curve with the maximum reduction of 6 could be seen for NIH-CPSI pain subscale (Figure 5B). However, the regression curves for NIH-CPSI urinary and QOL 
A

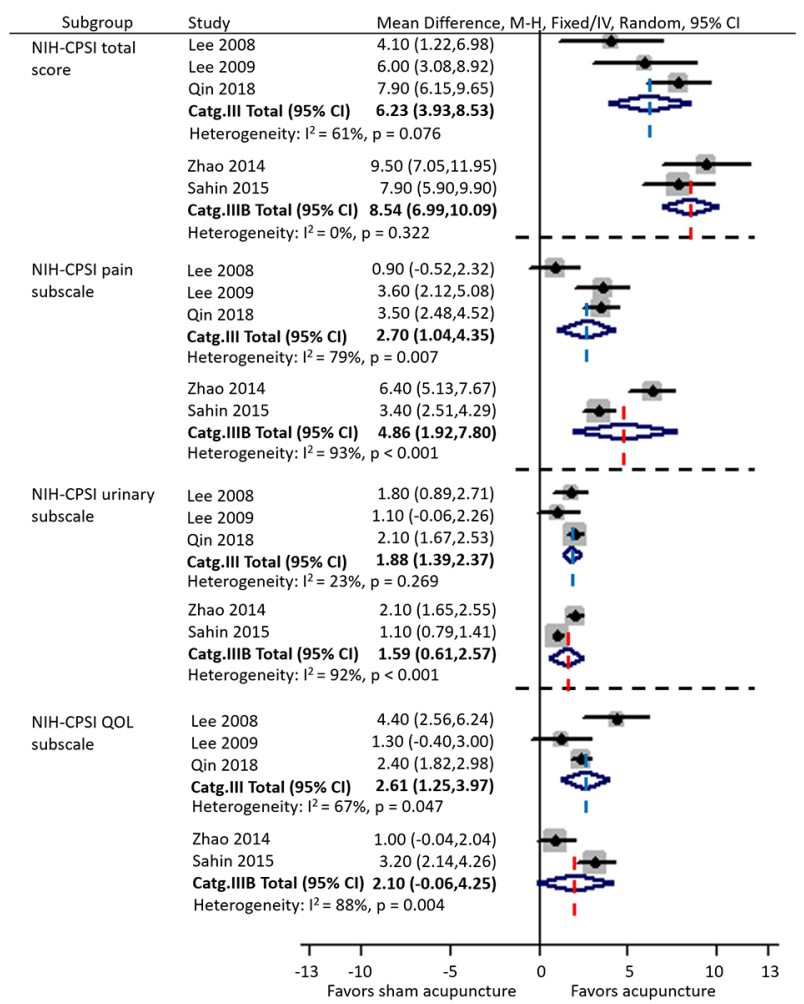

B

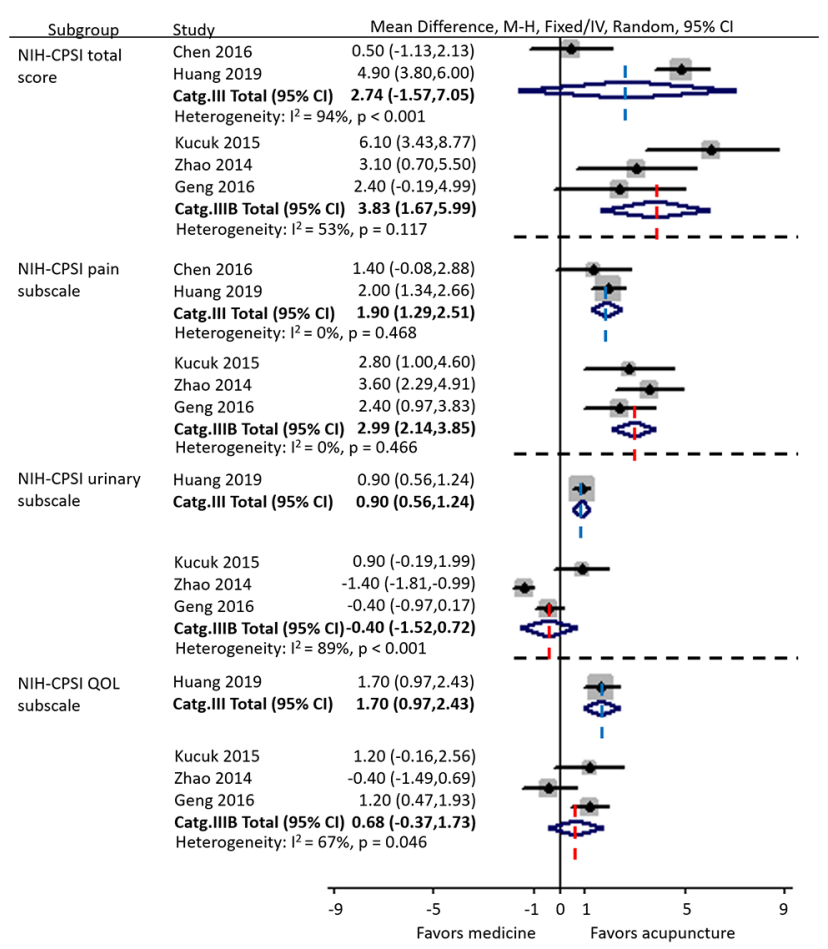

Figure 3 Subgroup forest plot of comparisons of NIH-CPSI score reduction by CP/CPPS categories. NIH-CPSI, National Institute of Health-Chronic Prostatitis Index; CP/CPPS, chronic prostatitis/chronic pelvic pain syndrome.

A Circular layout

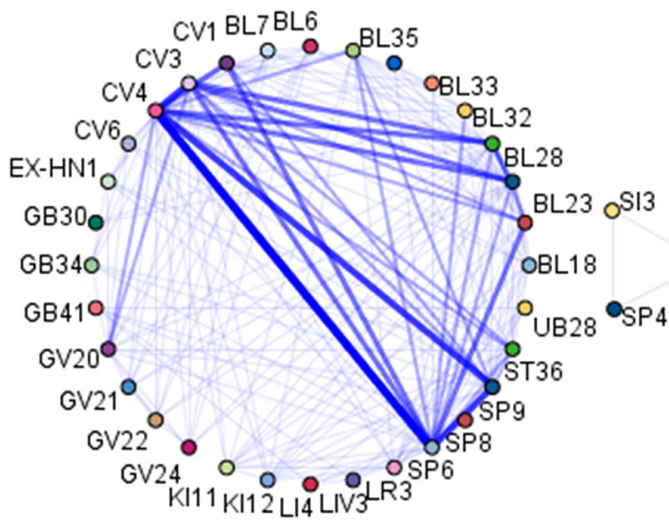

B Directional layout

OLU7

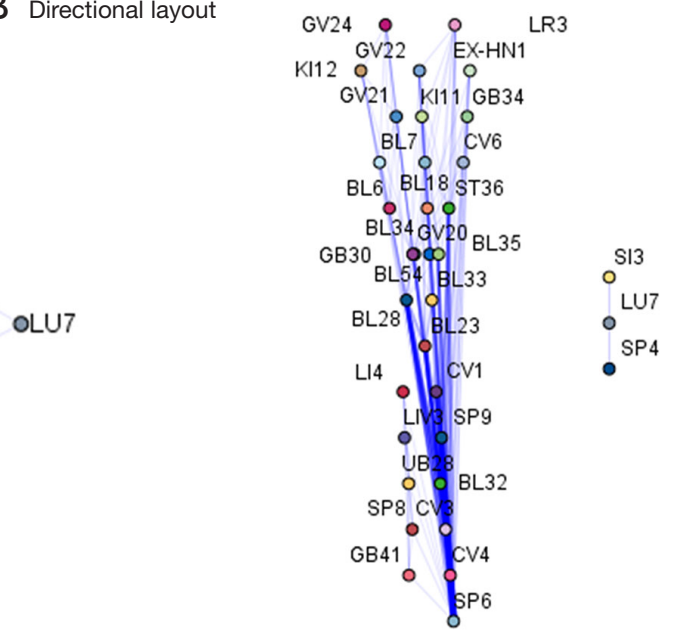

\section{OBL18 OBL23 OBL28 OBL32 ОвL33 ОвL34 OBL35 OBL54 OBL6 OBL7 OCV1 OCV3 OCV4 OCV6 OHN1 OGB30 OGB34 OGB41

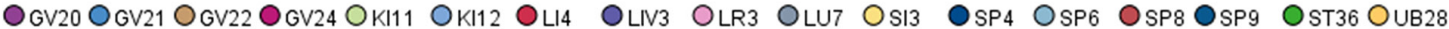

Figure 4 Apriori association analysis of acupoint selection. 

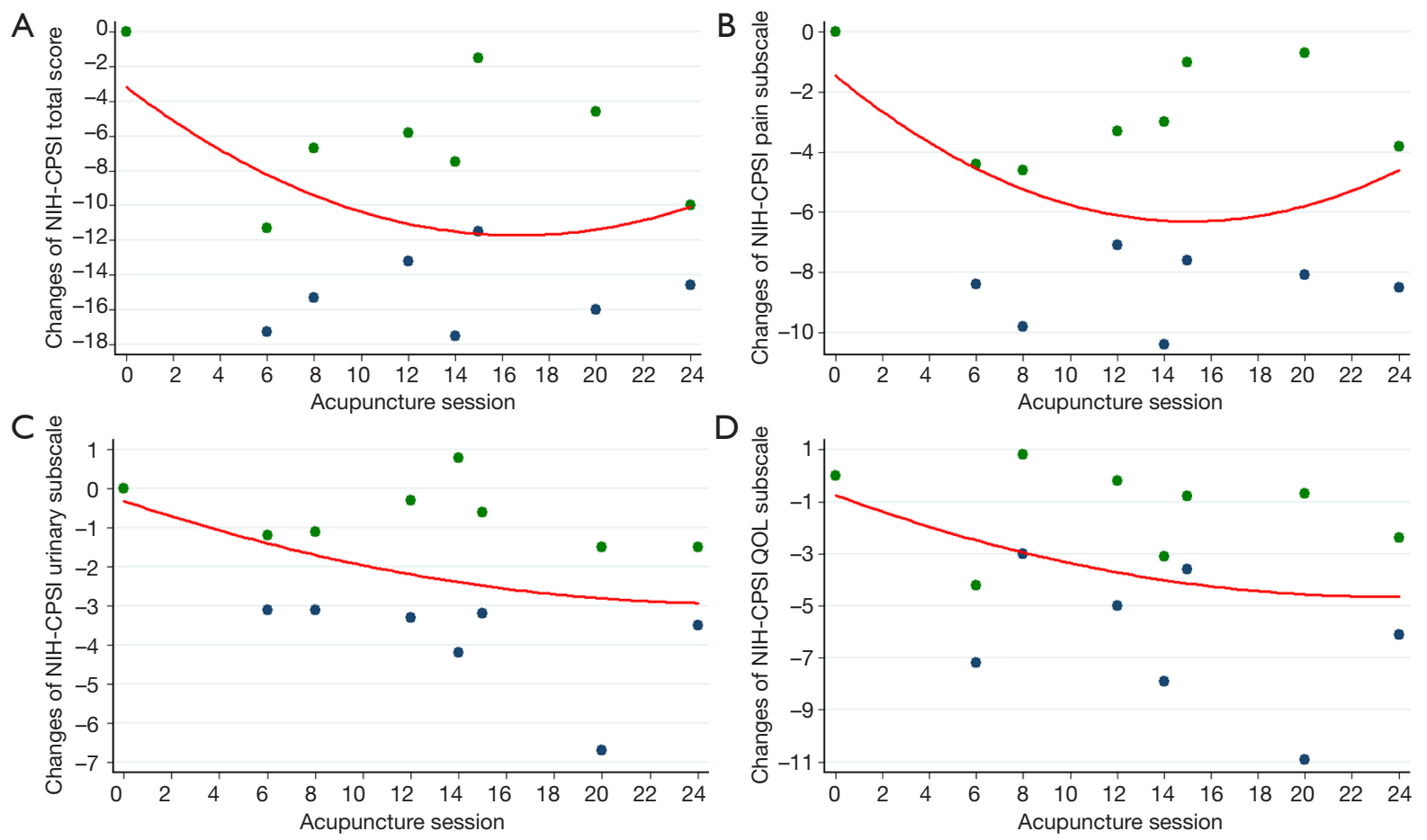

Figure 5 Dose-response relationship between acupuncture sessions and changes of NIH-CPSI score. NIH-CPSI, National Institute of Health-Chronic Prostatitis Index.

subscales showed that their scores kept declining with increasing acupuncture sessions, respectively reaching the maximum reduction of 3 and 5 after 24 acupuncture sessions (Figure 5C,D).

\section{Discussion}

Several pair-wise meta-analyses to assess acupuncture for CP/CPPS were previously conducted (23-25), and the evidence supported acupuncture as an effective treatment for CP/CPPS. Moreover, the network meta-analysis by Qin et al. also showed that acupuncture was the most effective in reducing the NIH-CPSI total score, followed by dual therapy of alpha-blockers and antibiotics, antibiotics, sham acupuncture and alpha-blockers (26). Our results confirmed that compared with sham acupuncture, acupuncture was a better treatment method for patients with CP/CPPS, in aspects of relieving pain and urinary symptoms and improving the QOL.

Since developed in 1999, NIH-CPSI has been widely used to rapidly assess the severity of CP/CPPS symptoms. This questionnaire provides an overall and valid assessment through covering the three most important symptom domains: pain, voiding and QOL (27). As the graded uniform outcome measure, NIH-CPSI standardizes measurement of CP/CPPS symptoms and allows more accurate comparisons between studies (28). On the contrary, the endpoint of response rate was limited because its defining standard varied among included trials. Thus, the NIH-CPSI score reduction not response rate was adopted as primary outcome in our study.

By meta-analysis to compare the efficacy on CP/ CPPS between acupuncture and medication (alphablockers and/or antibiotics), the study further indicated that acupuncture resulted in greater NIH-CPSI total score reduction and was better at relieving pain; but without medication related adverse effects such as nausea, dizziness, hypotension, and gastrointestinal symptoms. However, the results of previously published meta-analysis were inconsistent on this pair comparison, as some were in favor of medication (24) while some did not find any difference $(23,25)$. Possible causes were noted as follows. First, the drugs used (levofloxacin, tamsulosin, ibuprofen, celecoxib, Prostat, or their combination) were different among the trials. Second, different assessment criteria such as score reduction or post-treatment score were used in different 
trials. Third, the limited number of RCTs and their small sample size might be associated with the conflicting results. Furthermore, our meta-analysis indicated that combination therapy using acupuncture and medicine resulted in better improvement in NIH-CPSI total score than acupuncture or medicine monotherapy. This is consistent with the current evidence-based management of CP/CPPS, a multimodal therapeutic strategy because of a complex pathophysiology and heterogeneous clinical presentations for CP/CPPS (29). Although the small number of included trials limited the evidence level of pooled data, our study still suggests more benefits from the addition of acupuncture for patients' refractory to current medication.

Recent evidence suggested that the category IIIA and IIIB may represent two distinct pathological conditions or, alternatively, two different stages of the same condition (30), in that patients with category IIIA showed more severe signs and symptoms (NIH-CPSI scores and Qmax) than IIIB patients. Furthermore, the improvement of symptoms after medication was significantly more pronounced in IIIA patients when compared with IIIB patients. Thus, whether the differential response to acupuncture exists between IIIA and IIIB cohorts bothers us. Our subgroup meta-analysis suggested that compared with both sham acupuncture and medication, acupuncture appeared to be more effective in patients with category IIIB than category IIIA + IIIB, in aspects of NIH-CPSI total score and pain subscale. In addition, several recent meta-analysis demonstrated that antibiotic and its combination with alpha-blocker appeared to achieve the greatest improvement in clinical symptom scores compared with placebo $(31,32)$. The above results supported the opinion that category IIIA was caused by the pathogens. The possible reason is that some patients with bacterial prostatitis can be misdiagnosed as nonbacterial prostatitis due to local inflammatory obstruction or difficult-to-culture pathogens in prostatic duct $(16,33)$. The recently discovered nanobacterial infection was implicated to be an important etiologic factor of CP/CPPS $(34,35)$. In their study, anti-nanobacteria therapy could significantly improve symptoms of refractory CP/CPPS compared with placebo. Many urologists still thought the optimal treatment with antibiotics should be taken after CP/CPPS subtype evaluation.

Currently, uncertainty exists regarding the doseresponse relationship between acupuncture sessions and treatment efficacy on CP/CPPS, as clinical guidelines including acupuncture do not describe minimum treatment session in clinical practice. Facing the situation that the acupuncture sessions of included trials ranged from 6 to 28 times, we explored the dose-response relationship between acupuncture sessions and its efficacy. The overall trend suggested that more acupuncture sessions mean greater NIH-CPSI score reduction. Based on current evidence, four acupuncture sessions might be recommended as the minimum "dose" to reach its clinical efficacy. Thus, a short course of acupuncture treatment may be sufficient and effective in some cases, especially for acute pain. In addition, our data estimated that prolonged sessions of acupuncture had clinically different effects, especially continuously improved urinary symptoms and QOL. According to the population-based data from the National Health Interview Survey, most people who used acupuncture did not receive a full treatment course, which might affect the treatment efficacy (36). Thus, for refractory CP/CPPS, prolonged acupuncture sessions should be required for optimal efficacy.

In traditional Chinese medicine theory, individualized acupoint selection for each patient would be recommended according to physical examination (37). However, there were commonly used acupoints for CP/CPPS among different trials. Our data indicated that selection strategies of acupuncture points in most trials were on the basis of the combination of any three acupoints from CV3, CV4, BL32, SP6, and SP9. Although the exact therapeutic mechanism of acupuncture on CP/CPPS remains unclear, its beneficial therapeutic effects can be attributed to neuromodulation, immune-modulation, and anti-inflammatory efficacy. As shown in Table S2 and Figure 4, most trials chose the acupoints in the sacral area including CV3, CV4, and BL32. One explanation is that it acts on specific sacral nerves to modulate abnormal sacral reflex arcs, affecting the function of target organs or regions such as urethra pelvic floor (38). Besides peripheral neural pathways, acupuncture was also documented to modulate the activity of central neural pathways (39). This distal systemic effect may be attributed to the secretion of neurotransmitters such as endorphin, serotonin and dopamine in the brain stimulated by acupuncture to relieve pain and depression (40). It explained why some trials used auricular or other acupoints and still achieved therapeutic effects.

Our team previously investigated expressions of immune markers closely associated with autoimmunity including IgA, IgM, IgG, $\mathrm{CD}^{+}$and $\mathrm{CD}^{+}$in both CP/CPPS patients and healthy volunteers (41). We found that the CP/CPPS patients had lower serum $\mathrm{CD} 8^{+}$and higher serum $\mathrm{IgG}$ levels than healthy volunteers. It indicated that both cell immunity and humoral immunity might be involved in 
the development of CP/CPPS. Interestingly, the immune system may also be modulated by acupuncture. Lee et al. (42) revealed an increase in natural killer lymphocyte subpopulations after acupuncture, which was speculated to play a protective role in preventing CP/CPPS. Increasing evidences showed that acupuncture affected inflammatory mediators in patients with CP/CPPS. The levels of prostaglandin E2, TNF- $\alpha$ and IL- $1 \beta$ in prostatic fluid were demonstrated to be higher in CP/CPPS patients than normal populations, which could be significantly downregulated through acupuncture $(43,44)$.

Several limitations in our study that should be addressed. First, the quality assurance is very important in acupuncture clinical research or real-world practice. However, varied treatment protocols including different types and sessions of acupuncture, duration of each session, location of acupoints, and manipulation of the needle, may potentially impact clinical effects of acupuncture (45). The early work has been made to understand what aspects might constitute a quality acupuncture intervention (46-48). However, until now, no clearly reliable criteria or appraisal tools to assess the acupuncture quality has been developed (45). Second, the complexity of acupuncture also makes the differences amongst practitioners inevitable (47). Third, meta-analysis does not determine the specific patients may benefit from acupuncture due to the difficult of conducting subgroup analysis. Last, it is difficult to implement strictly doubleblind trials because of the features of acupuncture.

\section{Conclusions}

Acupuncture has promising efficacy for patients with CP/ CPPS, especially category IIIB, in aspects of relieving pain and urinary symptoms and improving the QOL. Compared to standard medication, acupuncture resulted in greater NIH-CPSI total score reduction and was better at relieving pain, but no differential improvement in urinary symptoms and QOL. Furthermore, better improvement in NIH-CPSI total score reduction preferred combination therapy using acupuncture and medication to acupuncture/medication monotherapy. As the minimum "dose" to reach clinical efficacy, four acupuncture sessions were recommended and prolonged sessions still continuously improved urinary symptoms and QOL.

\section{Acknowledgments}

Funding: This work was sponsored by National Major
Scientific and Technological Project for "Significant New Drugs Development" (2017ZX09304030) and Shanghai Sailing Program (18YF1422600). The funders had no role in study design, data collection and analysis, decision to publish, or preparation of the manuscript.

\section{Footnote}

Reporting Checklist: The authors have completed the PRISMA reporting checklist. Available at http://dx.doi. org/10.21037/tau-20-913

Peer Review File: Available at http://dx.doi.org/10.21037/ tau-20-913

Conflicts of Interest: All authors have completed the ICMJE uniform disclosure form (available at http://dx.doi. org/10.21037/tau-20-913). The authors have no conflicts of interest to declare.

Ethical Statement: The authors are accountable for all aspects of the work in ensuring that questions related to the accuracy or integrity of any part of the work are appropriately investigated and resolved.

Open Access Statement: This is an Open Access article distributed in accordance with the Creative Commons Attribution-NonCommercial-NoDerivs 4.0 International License (CC BY-NC-ND 4.0), which permits the noncommercial replication and distribution of the article with the strict proviso that no changes or edits are made and the original work is properly cited (including links to both the formal publication through the relevant DOI and the license). See: https://creativecommons.org/licenses/by-nc-nd/4.0/.

\section{References}

1. Krieger JN, Nyberg L, Jr., Nickel JC. NIH consensus definition and classification of prostatitis. JAMA 1999;282:236-7.

2. Engeler DS, Baranowski AP, Dinis-Oliveira P, et al. The 2013 EAU guidelines on chronic pelvic pain: is management of chronic pelvic pain a habit, a philosophy, or a science? 10 years of development. Eur Urol 2013;64:431-9.

3. Krieger JN, Riley DE, Cheah PY, et al. Epidemiology of prostatitis: new evidence for a world-wide problem. World J Urol 2003;21:70-4. 
4. Liang CZ, Li HJ, Wang ZP, et al. The prevalence of prostatitis-like symptoms in China. J Urol 2009;182:558-63.

5. Rees J, Abrahams M, Doble A, et al. Diagnosis and treatment of chronic bacterial prostatitis and chronic prostatitis/chronic pelvic pain syndrome: a consensus guideline. BJU Int 2015;116:509-25.

6. Herati AS, Moldwin RM. Alternative therapies in the management of chronic prostatitis/chronic pelvic pain syndrome. World J Urol 2013;31:761-6.

7. Cabyoglu MT, Ergene N, Tan U. The mechanism of acupuncture and clinical applications. Int J Neurosci 2006;116:115-25.

8. Blanchet KD. Acupuncture: gaining acceptance in urology: proves effective for a number of chronic conditions. BJU Int 2012;109:ii-iv.

9. Zhou M, Yang M, Chen L, et al. The effectiveness of long-needle acupuncture at acupoints BL30 and BL35 for CP/CPPS: a randomized controlled pilot study. BMC Complement Altern Med 2017;17:263.

10. Seong KM, Jang G, Kim DW, et al. Hwanglyunhaedok Pharmacopuncture versus Saline Pharmacopuncture on Chronic Nonbacterial Prostatitis/Chronic Pelvic Pain Syndrome. J Acupunct Meridian Stud 2017;10:245-51.

11. Qin Z, Zang Z, Zhou K, et al. Acupuncture for Chronic Prostatitis/Chronic Pelvic Pain Syndrome: A Randomized, Sham Acupuncture Controlled Trial. J Urol 2018;200:815-22.

12. Abrams KR, Gillies CL, Lambert PC. Meta-analysis of heterogeneously reported trials assessing change from baseline. Stat Med 2005;24:3823-44.

13. Lee SW, Liong ML, Yuen KH, et al. Acupuncture versus sham acupuncture for chronic prostatitis/chronic pelvic pain. Am J Med 2008;121:79.e1-7.

14. Lee SH, Lee BC. Electroacupuncture relieves pain in men with chronic prostatitis/chronic pelvic pain syndrome: three-arm randomized trial. Urology 2009;73:1036-41.

15. Sahin S, Bicer M, Eren GA, et al. Acupuncture relieves symptoms in chronic prostatitis/chronic pelvic pain syndrome: a randomized, sham-controlled trial. Prostate Cancer Prostatic Dis 2015;18:249-54.

16. Kucuk EV, Suceken FY, Bindayi A, et al. Effectiveness of acupuncture on chronic prostatitis-chronic pelvic pain syndrome category IIIB patients: a prospective, randomized, nonblinded, clinical trial. Urology 2015;85:636-40.

17. Huang JM, Deng C, Li X, et al. Effects of electroacupuncture combined with traditional chinese medicine fumigation on the efficacy of patients with chronic prostatitis and levels of IL-17 and TNF-a in prostatic fluid. Prog Modern Biomed 2019;19:1541-4.

18. Geng Q, Zhao Y, Ouyang B. The effectiveness of acupuncture for category IIIB chronic prostatitis/chronic pelvic pain syndrome. Lishizhen Medicine and Materia Medica Research 2016;27:1916-7.

19. Zhao JY, Song CS. Clinical observation of Jiaoxue acupuncturetreatment on IIIB prostatitis. The Chinese Journal of Human Sexuality 2014;23:57-9.

20. Chen G, Xiang J, Ouyang LZ, et al. Acupuncture combined with western medicine for CP/CPPS: a randomized controlled trial. Chinese Acupuncture and Moxibustion 2016;36:1247-51.

21. Yang MP, ZHang YJ. Clinical observation of acupuncture combined with western medicine for CP/CPPS. World Latest Medicine Information 2019;19:264-5.

22. Propert KJ, Litwin MS, Wang Y, et al. Responsiveness of the National Institutes of Health Chronic Prostatitis Symptom Index (NIH-CPSI). Qual Life Res 2006;15:299-305.

23. Qin Z, Wu J, Zhou J, et al. Systematic Review of Acupuncture for Chronic Prostatitis/Chronic Pelvic Pain Syndrome. Medicine (Baltimore) 2016;95:e3095.

24. Liu BP, Wang YT, Chen SD. Effect of acupuncture on clinical symptoms and laboratory indicators for chronic prostatitis/chronic pelvic pain syndrome: a systematic review and meta-analysis. Int Urol Nephrol 2016;48:1977-91.

25. Chang SC, Hsu CH, Hsu CK, et al. The efficacy of acupuncture in managing patients with chronic prostatitis/ chronic pelvic pain syndrome: A systemic review and metaanalysis. Neurourol Urodyn 2017;36:474-81.

26. Qin Z, Wu J, Tian J, et al. Network Meta-Analysis of the Efficacy of Acupuncture, Alpha-blockers and Antibiotics on Chronic Prostatitis/Chronic Pelvic Pain Syndrome. Sci Rep 2016;6:35737.

27. Wagenlehner FM, van Till JW, Magri V, et al. National Institutes of Health Chronic Prostatitis Symptom Index (NIH-CPSI) symptom evaluation in multinational cohorts of patients with chronic prostatitis/chronic pelvic pain syndrome. Eur Urol 2013;63:953-9.

28. Cohen JM, Fagin AP, Hariton E, et al. Therapeutic intervention for chronic prostatitis/chronic pelvic pain syndrome (CP/CPPS): a systematic review and metaanalysis. PLoS One 2012;7:e41941.

29. Magistro G, Wagenlehner FM, Grabe M, et al. Contemporary Management of Chronic Prostatitis/ Chronic Pelvic Pain Syndrome. Eur Urol 2016;69:286-97. 
30. Magri V, Marras E, Restelli A, et al. Multimodal therapy for category III chronic prostatitis/chronic pelvic pain syndrome in UPOINTS phenotyped patients. Exp Ther Med 2015;9:658-66.

31. Anothaisintawee T, Attia J, Nickel JC, et al. Management of chronic prostatitis/chronic pelvic pain syndrome: a systematic review and network meta-analysis. JAMA 2011;305:78-86.

32. Thakkinstian A, Attia J, Anothaisintawee T, et al. alphablockers, antibiotics and anti-inflammatories have a role in the management of chronic prostatitis/chronic pelvic pain syndrome. BJU Int 2012;110:1014-22.

33. Wagenlehner FM, Weidner W, Naber KG. Therapy for prostatitis, with emphasis on bacterial prostatitis. Expert Opin Pharmacother 2007;8:1667-74.

34. Shoskes DA, Thomas KD, Gomez E. Anti-nanobacterial therapy for men with chronic prostatitis/chronic pelvic pain syndrome and prostatic stones: preliminary experience. J Urol 2005;173:474-7.

35. Zhou Z, Hong L, Shen X, et al. Detection of nanobacteria infection in type III prostatitis. Urology 2008;71:1091-5.

36. Schwehr NA, Shippee ND, Johnson PJ. Acupuncture 'dose' (number of treatments) and insurance benefits in the USA. Acupunct Med 2018;36:88-95.

37. Lee SH, Lee BC. Use of acupuncture as a treatment method for chronic prostatitis/chronic pelvic pain syndromes. Curr Urol Rep 2011;12:288-96.

38. Shen J, Luo R, Zhang L, et al. Using electroacupuncture with optimized acupoint positioning to predict the efficacy of sacral neuromodulation of refractory overactive bladder: A case report. Medicine (Baltimore) 2019;98:e17795.

39. Zhou W, Benharash P. Effects and mechanisms of acupuncture based on the principle of meridians. J Acupunct Meridian Stud 2014;7:190-3.

Cite this article as: Zhang W, Fang Y, Shi M, Zhang M, Chen Y, Zhou T. Optimal acupoint and session of acupuncture for patients with chronic prostatitis/chronic pelvic pain syndrome: a meta-analysis. Transl Androl Urol 2021;10(1):143-153. doi: $10.21037 /$ tau-20-913
40. Lee SW, Liong ML, Yuen KH, et al. Validation of a sham acupuncture procedure in a randomised, controlled clinical trial of chronic pelvic pain treatment. Acupunct Med 2011;29:40-6.

41. Ye C, Xiao G, Xu J, et al. Differential expression of immune factor between patients with chronic prostatitis/ chronic pelvic pain syndrome and the healthy volunteers. Int Urol Nephrol 2018;50:395-9.

42. Lee SW, Liong ML, Yuen KH, et al. Acupuncture and immune function in chronic prostatitis/chronic pelvic pain syndrome: a randomized, controlled study. Complement Ther Med 2014;22:965-9.

43. Nadler RB, Koch AE, Calhoun EA, et al. IL-1beta and TNF-alpha in prostatic secretions are indicators in the evaluation of men with chronic prostatitis. J Urol 2000;164:214-8.

44. Shahed AR, Shoskes DA. Correlation of beta-endorphin and prostaglandin E2 levels in prostatic fluid of patients with chronic prostatitis with diagnosis and treatment response. J Urol 2001;166:1738-41.

45. Grant SJ, Smith CA, de Silva N, et al. Defining the quality of acupuncture: the case of acupuncture for cancer-related fatigue. Integr Cancer Ther 2015;14:258-70.

46. White AR, Ernst E. A trial method for assessing the adequacy of acupuncture treatments. Altern Ther Health Med 1998;4:66-71.

47. Price S, Long AF, Godfrey M, et al. Getting inside acupuncture trials--exploring intervention theory and rationale. BMC Complement Altern Med 2011;11:22.

48. Smith CA, Zaslawski CJ, Zheng Z, et al. Development of an instrument to assess the quality of acupuncture: results from a Delphi process. J Altern Complement Med 2011;17:441-52. 


\section{Supplementary}

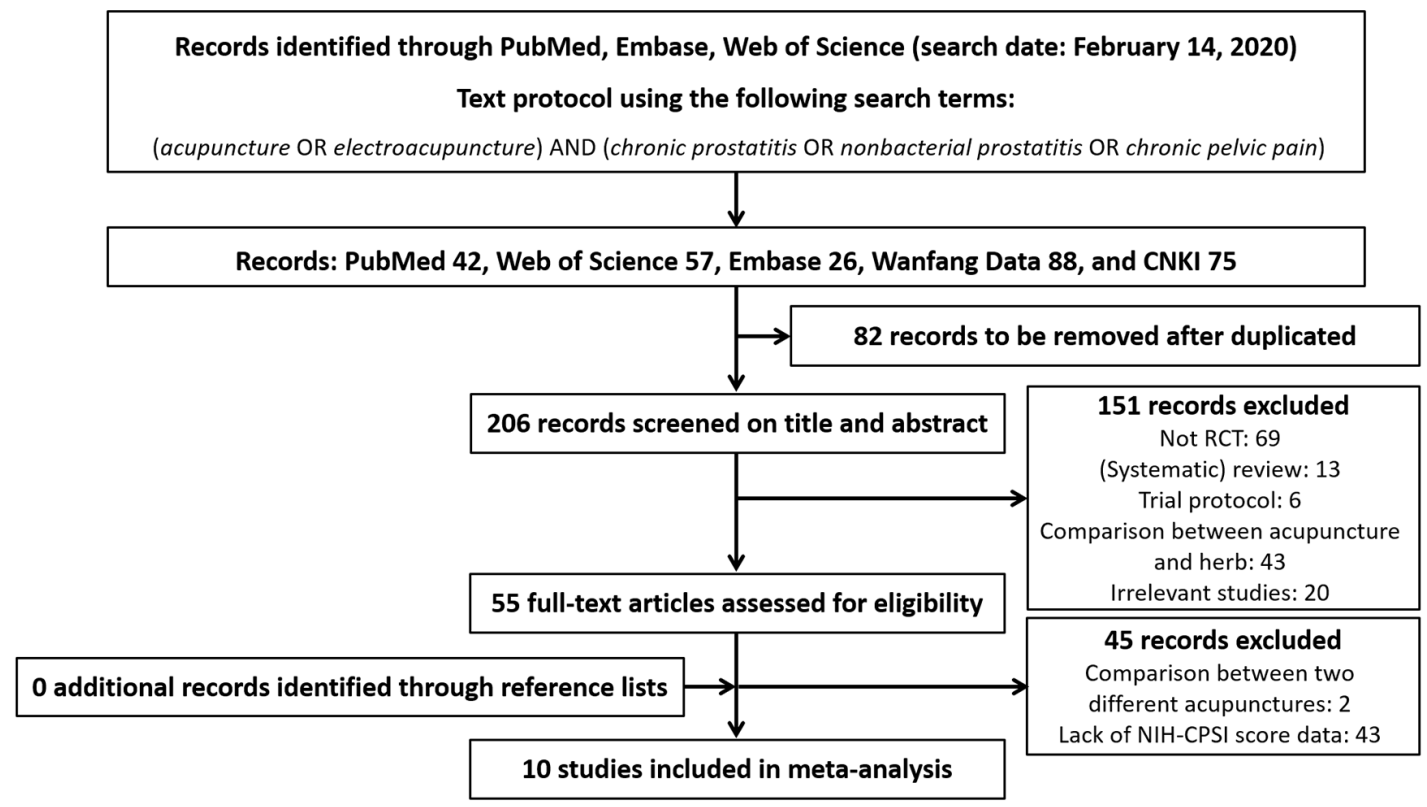

Figure S1 Flow diagram of search strategy.

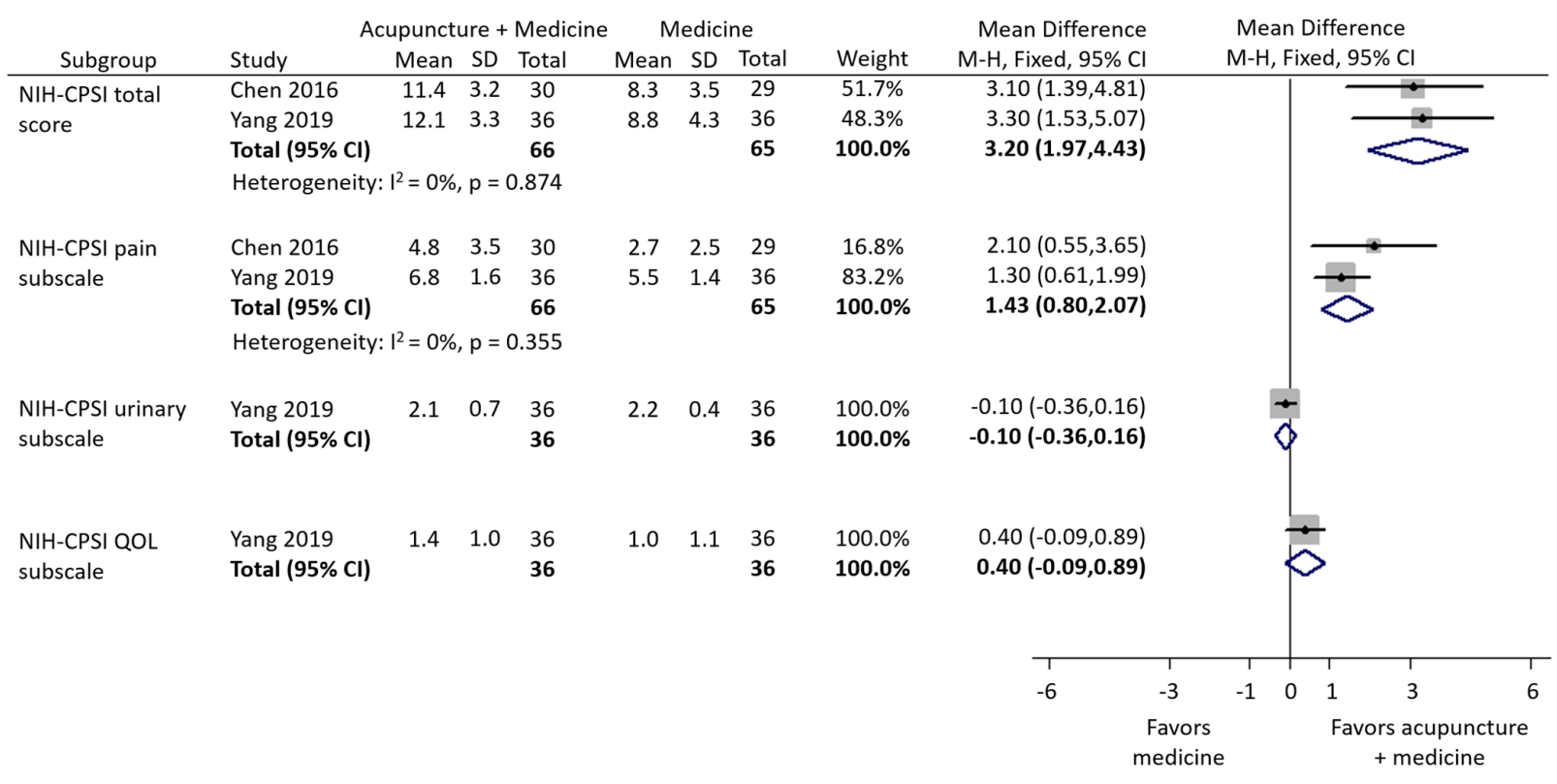

Figure S2 Forest plot of comparisons of NIH-CPSI score reduction between acupuncture combined with medication and medication monotherapy groups. NIH-CPSI, National Institute of Health-Chronic Prostatitis Index. 


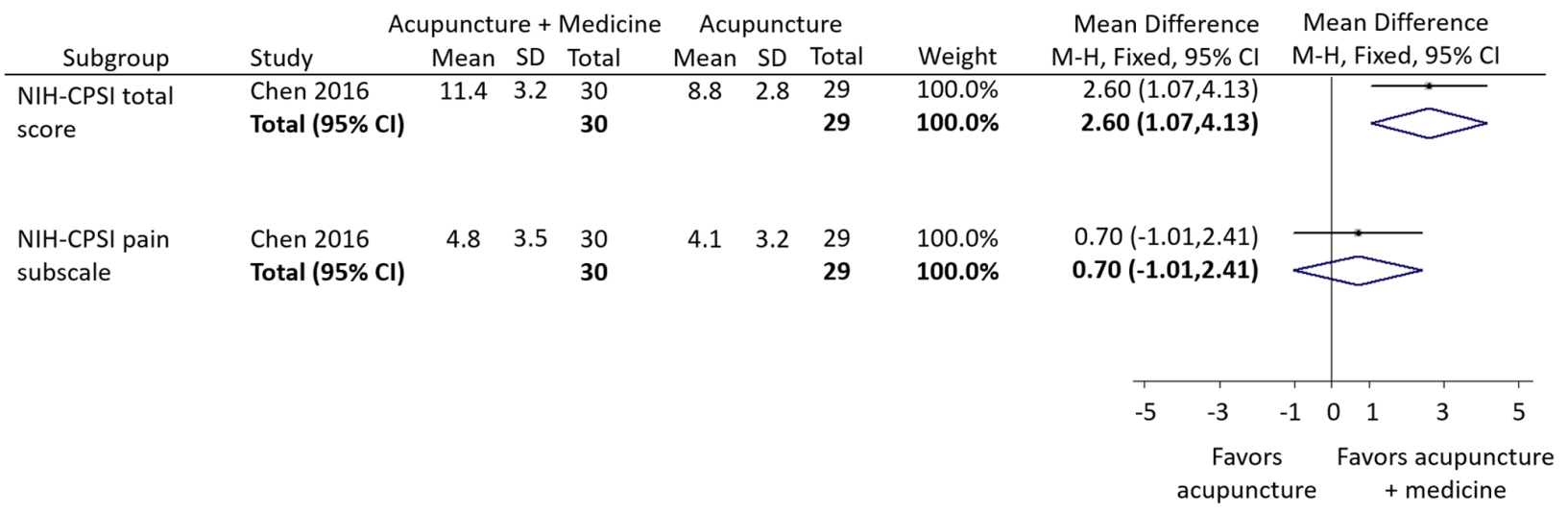

Figure S3 Forest plot of comparisons of NIH-CPSI score reduction between acupuncture combined with medication and acupuncture monotherapy groups. NIH-CPSI, National Institute of Health-Chronic Prostatitis Index.

Table S1 The baseline characteristics of the patients of the included studies

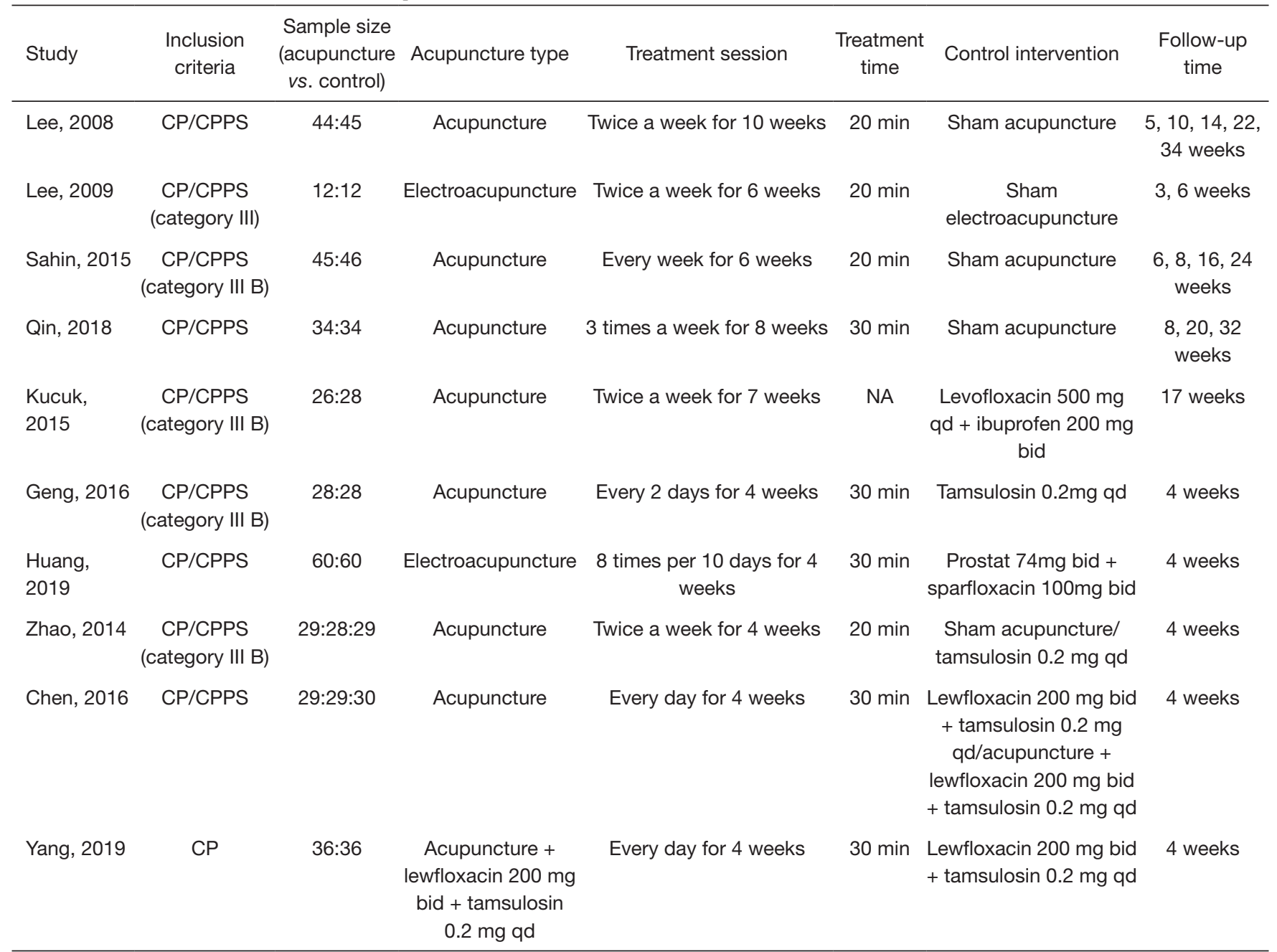

CP/CPPS, chronic prostatitis/chronic pelvic pain syndrome, min, minutes, mg, milligram, qd, quaque die (Latin), bid, bis in die (Latin). 
Table S2 The chosen acupoints of the included studies

\begin{tabular}{ll}
\hline Study & Acupoints \\
\hline Lee, 2008 & CV1 (Huiyin), CV4 (Guanyuan), SP6 (Saninjiao), SP9 (Yinlingquan) \\
Lee, 2009 & BL32 (Ciliao), BL33 (Zhongliao), GB30 (Huantiao) \\
Sahin, 2015 & BL33 (Zhongliao), BL34 (Xialiao), BL54 (Zhibian), CV1 (Huiyin), CV4 (Guanyuan), SP6 (Sanyinjiao), SP9 (Yinlingquan) \\
Qin, 2018 & BL23 (Shenshu), BL33 (Zhongliao), BL35 (Huiyang), SP6 (Sanyinjiao) \\
Kucuk, 2015 & UB28 (Pangguangshu), GB41 (Zulinqi), LIV3 (Taichong), LI4 (Hegu), SP6 (Sanyinjiao), SP8 (Diji) \\
Geng, 2016 & BL6 (Chengguang), BL7 (Tongtian), BL28 (Pangguangshu), BL32 (Ciliao), CV3 (Zhongji), CV4 (Guanyuan), GV20 (Baihui), \\
& GV21 (Qianding), GV22 (Xinhui), GV24 (Shenting) \\
Huang, 2019 & LR3 (Taichong), ST36 (Zusanli), KI11 (Henggu), KI12 (Dahe), CV3 (Zhongji), CV4 (Guanyuan), SP6 (Sanyinjiao), SP9 \\
& (Yinlingquan), BL18 (Ganshu), BL23 (Shenshu), BL28 (Pangguangshu), BL32 (Ciliao), BL54 (Zhibian) \\
Zhao, 2014 & LU7 (Lieque), SI3 (Houxi), SP4 (Gongsun) \\
Chen, 2016 & EX-HN1(Sishencong), GV20 (Baihui), CV3 (Zhongji), CV4 (Guanyuan), CV6 (Qihai), SP6 (Sanyinjiao), SP9 (Yinlingquan), \\
& GB34 (Yanglingquan), ST36 (Zusanli) \\
Yang, 2019 & CV1 (Huiyin), CV3 (Zhongji), CV4 (Guanyuan), BL23 (Shenshu), BL28 (Pangguangshu), BL32 (Ciliao), SP6 (Sanyinjiao) \\
\hline
\end{tabular}

